ANEXOS 

PUBLICACIONES DEL DEPARTAMENTO DE HISTORIA MEDIEVAL, CIENCIAS Y TÉCNICAS HISTORIOGRÁFICAS YESTUDIOS ÁRABES E ISLÁMICOS DE LA UNIVERSIDAD DE ZARAGOZA

\section{Revista Estudios de Edad Media de la Corona de Aragón}

$$
\begin{aligned}
1 & \text { (1945) } 344 \text { págs. } \\
2 & \text { (1946) } 580 \text { págs. } \\
3 & \text { (1948) } 776 \text { págs. } \\
4 & \text { (1951) } 744 \text { págs. } \\
5 & \text { (1952) } 853 \text { págs. } \\
6 & \text { (1956) } 553 \text { págs. } \\
7 & \text { (1962) } 832 \text { págs. } \\
8 & \text { (1967) } 808 \text { págs. } \\
9 & \text { (1973) } 736 \text { págs. } \\
10 & \text { (1975) } 840 \text { págs. }
\end{aligned}
$$

\section{Revista Aragón en la Edad Media}

1 (1977) Estudios de Economía y Sociedad en la Baja Edad Media, 276 págs.

2 (1979) Estudios de Economía y Sociedad (siglos XII al XV), 300 págs.

3 (1980) Estudios de Economía y Sociedad (siglos XII al XV), 262 págs.

4 (1981) Estudios de Economía y Sociedad (siglos XII al XV), 263 págs.

5 (1983) Estudios de Economía y Sociedad, 321 págs.

6 (1984) Estudios de Economía y Sociedad, 294 págs.

7 (1987) Estudios de Economía y Sociedad, 275 págs. 
8 (1989) Homenaje al profesor emérito Antonio Ubieto Arteta, 728 págs.

9 (1991) 496 págs.

10-11 (1993) Homenaje a la profesora emérita María Luisa Ledesma Rubio, 931 págs. 12 (1995) 340 págs.

13 (1997) 261 págs.

14-15 (1999) Homenaje a la profesora Carmen Orcástegui Gros, 2 vols., 1654 págs. 16 (2000) Homenaje al profesor emérito Ángel San Vicente Pino, 784 págs.

17 (2003) 300 págs.

18 (2004) 392 págs.

19 (2006) Homenaje a la profesora María Isabel Falcón Pérez, 629 págs.

20 (2008) Homenaje a la profesora María Desamparados Cabanes Pecourt, 868 págs

21 (2009) 332 págs.

22 (2011) 280 págs.

23 (2012) 312 págs.

24 (2013) 334 págs.

25 (2014) 372 págs.

26 (2015) 364 págs.

27 (2016) 348 págs.

28 (2017) 392 págs .

29 (2018) 250 págs.

30 (2019) 406 págs.

\section{Escuela de Estudios Medievales de Zaragoza (CSIC)}

Antonio Ubieto Arteta, Colección Diplomática de Pedro I de Aragón y de Navarra, Zaragoza, 1951, 514 págs.

M. Molho, El Fuero de Jaca. Edición crítica, Zaragoza, 1963, 664 págs.

Antonio Durán Gudiol, Colección diplomática de la Catedral de Huesca, Zaragoza, 1965 (vol. 1, 412 págs.) y 1969 (vol. 2, 516 págs.).

Pierre Tucoo-Chala, Cartulaires de la Vallée d'Ossau, Zaragoza, 1970, 425 págs.

José María Lacarra, El juramento de los Reyes de Navarra (1234-1329), Zaragoza, 1972.

Bonifacio Palacios Martín, La coronación de los Reyes de Aragón. 1204-1410. Aportación al estudio de las estructuras medievales, Valencia, 1975. 
José Ángel Sesma Muñoz y Esteban Sarasa Sánchez, Cortes del Reino de Aragón (1357-1451). Extractos y fragmentos de procesos desaparecidos, Valencia, 1976.

Pierre Tucoo-Chala, Le livre des hommages de Gaston Febus, Zaragoza, 1976.

María Isabel Falcón Pérez, Organización municipal de Zaragoza en el siglo XV, con notas acerca de los orígenes del régimen municipal en Zaragoza, Zaragoza, 1978 .

\section{Serie Varia del Departamento}

Estudios dedicados a Aragón de José María Lacarra. In memoriam, Zaragoza, 1987.

Quince temas medievales publicados por el profesor Don Antonio Ubieto, Zaragoza, 1991.

Juan F. Utrilla Utrilla y Germán Navarro Espinach (editores), Espacios de montaña. Las relaciones transpirenaicas en la Edad Media, Zaragoza, 2010.

Guillermo Tomás Faci, Libro de rentas y feudos de Ribagorza en 1322, Zaragoza, 2011.

Francisco Javier Gutiérrez González, Cerámica andalusí de la Seo de Zaragoza, Zaragoza, 2015.

\section{Actas de Seminarios de Doctorado de Historia Medieval}

Avances recientes en la historia del al-Andalus: arqueología y sociedad, anexo interno de la revista Aragón en la Edad Media, 9 (1991), 311-491.

Las sociedades de frontera en la España medieval, Zaragoza, 1993.

Paisajes rurales y paisajes urbanos: métodos de análisis en la historia medieval, Zaragoza, 1993.

Violencia y conflictividad en la sociedad de la España bajomedieval, Zaragoza, 1995.

El Estado en la Baja Edad Media: nuevas perspectivas metodológicas, Zaragoza, 1999.

Sociedad, cultura e ideologías en la España bajomedieval, Zaragoza, 2000.

Rentas, producción y consumo en España en la Baja Edad Media, Zaragoza, 2001.

Demografía y sociedad en la España bajomedieval, Zaragoza, 2002.

Perspectivas actuales sobre las fuentes notariales de la Edad Media, Zaragoza, 2004.

Pescar o navegar: la Edad Media en la red, Zaragoza, 2005.

La prosopografía como método de investigación sobre la Edad Media, Zaragoza, 2006.

Familia y sociedad en la Edad Media (siglos XII-XV), Zaragoza, 2007. 


\section{Colección de Textos e Instrumentos para la Investigación}

María Isabel Falcón Pérez, Prosopografía de los infanzones de Aragón (1200-1400), Zaragoza, 2003.

Ángel J. Martín Duque, Colección Diplomática del Monasterio de San Victorián de Sobrarbe (1000-1219), Zaragoza, 2004.

José Ángel Sesma Muñoz, Huesca, ciudad de mercado de ámbito internacional en la Baja Edad Media según sus registros de aduana, Zaragoza, 2005.

José Ángel Sesma Muñoz, La vía del Somport en el comercio medieval de Aragón (Los registros de las aduanas de Jaca y Canfranc de mediados del siglo XV), Zaragoza, 2006.

Carlos Laliena Corbera y Eric Knibbs, El cartulario del monasterio aragonés de San Andrés de Fanlo (siglos X-XIII), Zaragoza, 2007.

María Teresa Iranzo Muñío, Política municipal y vida pública en Huesca. Documentos (1260-1527), Zaragoza, 2008.

Germán Navarro Espinach, Cuentas del Concejo de Mirambel (1472-1489), Zaragoza, 2008.

Germán Navarro Espinach y Concepción Villanueva Morte, Libro de la Bailía de Cantavieja (1428-1470), Zaragoza, 2009.

Sandra de la Torre Gonzalo, El cartulario de la encomienda templaria de Castellote (Teruel), 1184-1283, Zaragoza, 2009.

José Ángel Sesma Muñoz, El tráfico mercantil por las aduanas de Ribagorza (14441450). Producción y comercio rural en Aragón a finales de la Edad Media, Zaragoza, 2010.

José Ángel Sesma Muñoz y Juan Abella Samitier, Las aduanas de las Cinco Villas a mediados del siglo $\mathrm{XV}$, Zaragoza, 2013.

Santiago Simón Ballesteros, Documentos sobre la segunda unión aragonesa (13471348). Colección procedente del Archivo de la Corona de Aragón, Zaragoza, 2015.

Concepción Villanueva Morte y Mario Lafuente Gómez (coords.), Documentos del Concejo de Zaragoza. Edición crítica de los fondos del Archivo Municipal. I (1285-1348), Zaragoza, 2015.

\section{Grupo de Investigación CEMA}

(Centro de Estudios Medievales de Aragón)

\section{Colección Acta Curiarum Regni Aragonum}

Tomo 1 - Jaime I, Pedro III, Alfonso III, Jaime II y Alfonso IV (En preparación). 
Tomo 2 - Cortes y parlamentos del reinado de Pedro IV/1, edición a cargo de José Ángel Sesma Muñoz y Mario Lafuente Gómez, Zaragoza, 2013:

1336 - Cortes de Zaragoza y Parlamento de Gandesa.

1347 - Cortes de Zaragoza.

1348 - Cortes de Zaragoza.

1349 - Cortes de Zaragoza.

1350 - Cortes de Zaragoza.

1352 - Cortes de Zaragoza.

1354 - Parlamento de Alcañiz.

1356 - Cortes de Daroca.

1357 - Cortes de Cariñena.

1360 - Cortes de Zaragoza.

1361 - Parlamento de Cariñena.

1362 - Parlamento de Barbastro.

1362/1363 - Cortes Generales de Monzón.

1364/1365 - Cortes de Zaragoza.

Tomo 3 - Cortes del reinado de Pedro IV/2, edición a cargo de Carlos Laliena Corbera, Zaragoza, 2008:

1365/1366 - Cortes de Zaragoza y Calatayud.

1367 - Cortes de Zaragoza.

1371/1372 - Cortes de Caspe, Alcañiz y Zaragoza.

1375 - Cortes de Tamarite de Litera.

Tomo 4 - Cortes del reinado de Pedro IV/3, edición a cargo de José Ángel Sesma Muñoz, Zaragoza, 2006:

1375/1376 - Cortes Generales de Monzón

Tomo 5 - Cortes del reinado de Pedro IV/4 y Juan I, edición a cargo de José Ángel Sesma Muñoz, Zaragoza, 2009:

1381 - Cortes de Zaragoza.

1383/1384 - Cortes Generales de Monzón, Tamarite de Litera y Fraga.

1388/1389 - Cortes Generales de Monzón.

Tomo 6 (2 vols.) - Cortes del reinado de Martín I, edición a cargo de Germán Navarro

Espinach, Zaragoza, 2008:

1398/1400 - Cortes de Zaragoza.

1404 - Cortes de Maella.

1405 - Fogaje General de Aragón. 
Tomo 7 (2 vols.) - Parlamentos del Interregno, edición a cargo de José Ángel Sesma Muñoz, Zaragoza, 2011:

1411/1412 - Parlamento de Alcañiz y Zaragoza.

1412 - Compromiso de Caspe.

1412/06/28 - Sentencia del Compromiso de Caspe.

Tomo 8 - Cortes del reinado de Fernando I, edición a cargo de Germán Navarro Espinach, Zaragoza, 2009:

1412 - Cortes de Zaragoza.

1413/1414 - Cortes de Zaragoza.

Tomo 9 (2 vols.) - Cortes del reinado de Alfonso V/1, edición a cargo de María Teresa Iranzo Muñío, Zaragoza, 2007:

1423 - Cortes de Maella.

1427/1428 - Cortes de Teruel.

1429 - Cortes de Valderrobres.

1435 - Cortes Generales de Monzón.

1436 - Cortes de Alcañiz.

Tomo 10 (3 vols.) - Cortes del reinado de Alfonso V/2, edición a cargo de Carlos Laliena Corbera y María Teresa Iranzo Muñío, Zaragoza, 2016.

1439 - Cortes de Zaragoza.

1441/1442 - Cortes de Alcañiz-Zaragoza.

1442 - Cortes de Zaragoza.

1446/1450 - Cortes de Zaragoza.

Tomo 11 (2 vols.) - Cortes del reinado de Alfonso V/3, edición a cargo de Guillermo Tomás Faci, Zaragoza, 2013:

1451/1454 - Cortes de Zaragoza.

Tomo 12 - Juan II (En preparación):

1460 - Cortes de Fraga.

1461 - Cortes de Calatayud.

1467 - Cortes de Zaragoza.

1469/1470 - Cortes Generales de Monzón.

Tomo 13 - Cortes del reinado de Fernando II/I, edición a cargo de Cristina Monterde, José Ángel Sesma y Blanca Ferrez, Zaragoza, 2019:

1484 - Cortes Generales de Tarazona.

1488 - Cortes de Zaragoza.

1493 - Cortes de Zaragoza. 
Tomo 14 - Fernando II (En preparación):

1495/1496 - Cortes de Tarazona y Fogaje General del Reino.

Tomo 15 (2 vols.) - Cortes del reinado de Fernando II/3, edición a cargo de José Ángel

Sesma y Carlos Laliena, Zaragoza, 2017:

1498 - Cortes de Zaragoza.

1502 - Cortes de Tarazona.

Tomo 16 (2 vols.) - Cortes del reinado de Fernando II/4, edición a cargo de Cristina

Monterde Albiac, Zaragoza, 2011:

1510 - Cortes Generales de Monzón.

1512/1514 - Cortes Generales de Monzón.

COLECCIÓN GARBA. Colectánea de estudios sobre temas de Historia Medieval

José Ángel Sesma Muñoz y Carlos Laliena Corbera (coords.), La población de Aragón en la Edad Media (siglos XII-XV). Estudios de demografía histórica, Zarago- za, 2004.

José Ángel Sesma Muñoz y Carlos Laliena Corbera (coords.), La pervivencia del concepto. Nuevas reflexiones sobre la ordenación social del espacio en la Edad Media, Zaragoza, 2008.

José Ángel Sesma Muñoz y Carlos Laliena Corbera (coords.), Crecimiento económico y formación de los mercados en Aragón en la Edad Media (1200-1350), Zaragoza, 2009.

José Ángel Sesma Muñoz (coord.), La Corona de Aragón en el centro de su historia (1208-1458). La Monarquía aragonesa y los reinos de la Corona, Zaragoza, 2010.

José Ángel Sesma Muñoz (coord.), La Corona de Aragón en el centro de su historia (1208-1458). Aspectos económicos y sociales, Zaragoza, 2010.

José Ángel Sesma Muñoz (coord.), La Corona de Aragón en el centro de su historia (1208-1458). El Interregno y el Compromiso de Caspe (1410-1412), Zaragoza, 2012. Carlos Laliena Corbera y Mario Lafuente Gómez (coords.), Una economía integrada. Comercio, instituciones y mercados en Aragón, 1300-1500, Zaragoza, 2012.

José Ángel Sesma Muñoz y Carlos Laliena Corbera (coords.), De la escritura a la Historia (Aragón, siglos XIII-XV). Estudios dedicados a la profesora Cristina Monterde Albiac, Zaragoza, 2014.

Carlos Laliena Corbera y Mario Lafuente Gómez (coord.), Consumo, comercio y transformaciones culturales en la Baja Edad Media: Aragón, siglos XIV-XV, Zaragoza, 2016. 


\section{Colección MAnCuso. Monografías de Historia Medieval con apéndice documental}

María Teresa Iranzo Muñío, La peripecia del puente de piedra de Zaragoza durante la Edad Media, Zaragoza, 2005.

Carlos Laliena y Julián Ortega, Arqueología y poblamiento. La cuenca del río Martín en los siglos V-VIII, Zaragoza, 2005.

José Ángel Sesma Muñoz, Los idus de diciembre de Fernando II. El atentado del rey de Aragón en Barcelona, Zaragoza, 2006.

Enrique Mainé Burguete, Ciudadanos honrados de Zaragoza. La oligarquía zaragozana en la Baja Edad Media (1370-1410), Zaragoza, 2006.

María Luis Cercós Vallés y Javier Medrano Adán, Mirambel entre dos mundos. Paisaje y comunidad en el Maestrazgo a fines de la Edad Media, Zaragoza, 2011.

Mario Lafuente Gómez, Dos Coronas en guerra. Aragón y Castilla (1356-1366), Zaragoza, 2012.

Sandra de la Torre Gonzalo, Construir el paisaje. Hábitat disperso en el Maestrazgo turolense de la Edad Media, Zaragoza, 2012.

\section{Área de Estudios Árabes e Islámicos}

Revista Estudios de Dialectología Norteafricana y Andalusí (EDNA)

1 (1996) 245 págs.

2 (1997) 249 págs.

3 (1998) 229 págs.

4 (1999) 247 págs.

5 (2000-2001) 313 págs.

6 (2002) 273 págs.

7 (2003) 213 págs.

8 (2004) 296 págs. Homenaje a Peter Behnstedt en su 60 aniversario.

9 (2005) 290 págs.

10 (2006) 297 págs.

11 (2007) 185 págs.

12 (2008) 155 págs.

13 (2009) 265 págs. Special Issue: Women's World-Women's Word: Female life as reflected in the Arabic dialects.

\section{Colección Área de Estudios Árabes e Islámicos}

Corriente, Federico, Léxico estándar y andalusí del Dîwân de Ibn Quzmân. Zaragoza: Universidad de Zaragoza, 1993. 
Ferrando, Ignacio, 23 contratos comerciales escritos por los judios de Toledo en los siglos XIII y XIV: edición completa y estudio lingüístico de los datos judeo-árabes y andalusíes. Zaragoza: Universidad de Zaragoza, 1994.

Corriente, Federico y Bouzineb, Hussain, Recopilación de refranes andalusíes de Alonso del Castillo. Zaragoza: Universidad de Zaragoza, 1994.

Ferrando, Ignacio, El dialecto andalusí de la marca media: los documentos mozárabes toledanos de los siglos XII y XIII. Zaragoza: Universidad de Zaragoza, 1995.

Ould Mohamed Baba, Ahmed-Salem, Estudio dialectológico y lexicológico del refranero andalusí de Abû Yahyà Azzajjâlî. Zaragoza: Universidad de Zaragoza, 1999.

Vicente, Ángeles, El dialecto árabe de Anjra (norte de Marruecos). Estudio lingüístico y textos. Zaragoza: Universidad de Zaragoza, 2000.

Abu-Shams, Leila, Estudio lingüístico y textual del léxico relativo a la cocina y la alimentación en el dialecto árabe de Rabat (Marruecos). Zaragoza: Universidad de Zaragoza, 2002.

\section{Actas de Congresos}

Aguadé, Jordi, Corriente, Federico, Marugán, Marina (eds.), Actas del congreso internacional sobre interferencias lingüísticas arabo-romances y paralelos extraiberos. Zaragoza, 1994.

Aguadé, Jordi, Cressier, Patrice, Vicente, Ángeles (eds.), Peuplement et arabisation au Maghreb occidental. Dialectologie e histoire. Madrid-Zaragoza: Casa de Ve- lázquez-Universidad de Zaragoza, 1998.

\section{Colección Estudios de Dialectología Árabe}

Federico Corriente y Ángeles Vicente (con la colaboración de F. Abu-Haidar, J. Aguadé, P. Behnstedt, J. Dickins, O. Jastrow, A.-S. Ould Mohamed-Baba, P. Sánchez, M. Vanhove, A. Zaborski). Manual de dialectología neoárabe. Zaragoza: Instituto de Estudios Islámicos y del Oriente Próximo, 2008.

Ahmed-Salem Ould Mohamed-Baba, Refranero y fraseología hassān̄̄. Recopilación explicación, estudio gramatical y glosario. Zaragoza: Instituto de Estudios Islámicos y del Oriente Próximo, 2008.

Simon Lévy, Parlers arabes des Juifs du Maroc. Histoire, sociolinguistique et géographie dialectale.Zaragoza: Instituto de Estudios Islámicos y del Oriente Próxi- mo, 2009.

Christophe Pereira, Le parler árabe de Tripoli (Libye). Zaragoza: Instituto de Estudios Islámicos y del Oriente Próximo, 2010.

Montserrat Benítez Fernández, La política lingüística contemporánea de Marruecos: de la arabización a la aceptación del multilingüismo. Zaragoza: Instituto de Estudios Islámicos y del Oriente Próximo, 2010.

Mohamed Meouak, Pablo Sánchez, Ángeles Vicente (eds.) De los manuscritos medie- 
vales a internet: la presencia del árabe vernáculo en las fuentes escritas. Zaragoza: Universidad de Zaragoza, 2012.

Alexandrine Barontini, Christophe Pereira, Ángeles Vicente, Karima Ziamari (eds.), Dynamiques langagières en Arabophonies: variations, contacts, migrations et créations artistiques. Hommage offert à Dominique Caubet par ses elèves et collègues. Zaragoza: Universidad de Zaragoza / INaLCO, 2012.

Pablo Sánchez, El árabe vernáculo de Marrakech: análisis lingüístico de un corpus representativo. Zaragoza: Prensas de la Universidad de Zaragoza, 2014.

Montserrat Benítez Fernández (ed.), Trabajo y palabra: dos formas de expresión de las mujeres de Marruecos. Zaragoza: Prensas de la Universidad de Zaragoza, 2014.

Jairo Guerrero Parrado, El dialecto árabe hablado en la ciudad marroquí de Larache. Zaragoza: Prensas de la Universidad de Zaragoza, 2015.

Carmen Berlinches, El dialecto árabe de Damasco (Siria): estudio gramatical y textos. Zaragoza: Prensas de la Universidad de Zaragoza, 2016.

Ángeles Vicente, Dominique Caubet, Amina Naciri-Azzouz (éds). La région du NordOuest marocain : Parlers et pratiques sociales et culturelles. Zaragoza: Prensas de la Universidad de Zaragoza, 2017.

Veronika Ritt-Benmimoun, Tunisian and Libyan Arabic Dialects. Common trends Recent developments - Diachronic aspects. Zaragoza: Prensas de la Universidad de Zaragoza, 2017.

Laura Gago Gómez, Aproximación a la situación sociolingüística de Tánger-Arcila: variación léxica y grafemática. Zaragoza: Prensas de la Universidad de Zaragoza, 2018.

Letizia Lombezzi, Contents and Methods for Teaching Spoken Arabic. Zaragoza: Prensas de la Universidad de Zaragoza, 2019.

Emanuela de Blasio, Il rap nel mundo arabo: una forma d'avanguardia. Analisi di un corpus di area vicinorientale. Zaragoza: Prensas de la Universidad de Zaragoza, 2019.

\section{Serie Estudios Árabes e Islámicos (IEIOP)}

Ibn Hayyân, Crónica de los emires Alhakam I y 'Abderrahman II entre los años 796 y 847 [Almuqtabis II-1]. Mahmud Ali Makki y Federico Corriente Córdoba (trads.). Zaragoza: Instituto de Estudios Islámicos y del Oriente Próximo, 2001.

Ángeles Vicente (ed.). Musulmanes en el Aragón del siglo XXI. Zaragoza: Instituto de Estudios Islámicos y del Oriente Próximo, 2004.

Aly Aben Ragel, El Libro Conplido en los Iudizios de las Estrellas. Partes 6 a 8. Zaragoza: Instituto de Estudios Islámicos y del Oriente Próximo, 2005.

Vicent Barletta, Gestos Clandestinos: la literatura aljamiado-morisca como práctica cultural. Zaragoza: Instituto de Estudios Islámicos y del Oriente Próximo, 2005. 
Alberto Montaner Frutos y Alfonso Boix Jovaní, Guerra en Sarq al-Andalus: las batallas cidianas de Morella (1084) y Cuarte (1094). Zaragoza: Instituto de Estudios Islámicos y del Oriente Próximo, 2005.

Jordi Aguadé, Ángeles Vicente y Leila Abushams (eds.). Sacrum Arabo - Semiticum. Homenaje al prof. Federico Corriente en su 65 aniversario. Zaragoza: Instituto de Estudios Islámicos y del Oriente Próximo, 2005.

Christy Bandak, Libros de los buenos proverbios. Estudio y edición crítica de las versiones castellana y árabe. Zaragoza: Instituto de Estudios Islámicos y del Oriente Próximo 2007.

George T. Beech, The brief eminence and doomed fall of Islamic Saragossa. A great center of Jewish and Arabic learning in the Iberian Peninsula during the $11^{\text {th }}$ century. Zaragoza: Instituto de Estudios Islámicos y del Oriente Próximo, 2008.

Hans-Jörg Döhla, El libro de Calila e Dimna. Nueva edición y estudio de los dos manuscritos castellanos. Zaragoza: Instituto de Estudios Islámicos y del Oriente Próximo, 2009.

Nuria Martínez de Castilla Muñoz, Una biblioteca morisca entre dos tapas. Zaragoza: Instituto de Estudios Islámicos y del Oriente Próximo, 2010. 



\section{NORMAS GENERALES PARA LA PRESENTACIÓN DE ORIGINALES}

La revista Aragón en la Edad Media es una publicación digital (e-ISSN: $2387-$ 1377) e impresa (ISSN: 0213-2486) de periodicidad anual que recoge investigaciones originales o balances historiográficos inéditos sobre la historia medieval de cualquiera de los antiguos territorios de la Corona de Aragón. El consejo editorial está formado por especialistas reconocidos en la materia de ámbito internacional. Se aceptan artículos en castellano, catalán, inglés, francés, italiano y portugués. La revista está indexada en múltiples bases de datos: DICE, RESH, MIAR, ISOC, Regesta Imperii, Latindex, Dialnet, Repertorio de Medievalismo Hispánico, etc.

Los originales se envían a través de la plataforma Open Journal System de la Universidad de Zaragoza: <https://papiro.unizar.es/ojs/index.php/aem> Cualquier consulta puede dirigirse al correo <aragonem@unizar.es>. La revista se reserva el derecho de rechazar cualquier original que, por criterios formales o de calidad, considere que no es necesario que inicie el proceso de evaluación. Dicha decisión se comunicará a los autores en un plazo máximo de un mes desde la fecha de recepción. No se admiten estudios que, simultáneamente, se hallen en proceso de evaluación para su edición en otra revista científica. Cuando la dirección de la revista confirme la admisión a trámite de un artículo éste pasará a ser revisado por al menos dos evaluadores externos. En un plazo máximo de tres meses desde la fecha de recepción se informará sobre la aceptación o rechazo del mismo. Si los artículos superan el proceso de evaluación, los autores deberán incorporar, en su caso, las modificaciones sugeridas por los evaluadores. Además, deberán adaptar escrupulosamente los originales al formato requerido por la revista. De otro modo, la dirección se reserva el derecho de devolvérselos a los autores y no publicarlos hasta que lo cumplan. Cuando un texto haya sido profundamente reestructurado podrá ser sometido a un nuevo proceso de evaluación para confirmar su edición. Las primeras pruebas de impresión de los artículos siempre serán corregidas por la dirección de la revista. Los derechos de edición corresponden a Prensas de la Universidad de Zaragoza. Finalmente, los autores recibirán una separata del artículo en formato pdf.

Los artículos no podrán superar la extensión de 95.000 caracteres con espacios (notas, espacios y bibliografía incluidos). Después del título en el idioma original del artículo se incluirá su traducción al inglés. A continuación se indicará el nombre y apellidos del autor y correo electrónico de contacto, debajo, la institución en la que desarrolla sus actividades en el idioma original. Los estudios irán precedidos de sendos resúmenes de entre cinco y diez líneas en castellano y en inglés (abstract). 
Asimismo, se incluirán de tres a cinco palabras clave (keywords) en castellano e inglés. Entre los dos resúmenes y sus respectivas palabras clave, no se superarán nunca los 1500 caracteres con espacios incluidos. Deberá constar explícitamente el título del trabajo (preciso y breve, conteniendo el mayor número posible de palabras clave). Si es muy largo, se recomienda la división en título y subtítulo.

Por lo que respecta al texto, se remitirá preferentemente en formato Word en un único documento o archivo; en caso contrario, se indicará en hoja aparte el nombre de cada documento y su orden. Para evitar confusiones, los originales habrán de presentarse con las páginas numeradas de forma correlativa. El interlineado, los márgenes, tipo de letra y otras características de formato serán uniformes, con la única excepción de las citas extensas, que llevarán sangría mayor y un cuerpo de letra menor. Las citas que no vayan en párrafo aparte se presentarán entrecomilladas, nunca en cursiva.

Las llamadas de nota irán tras el signo de puntuación cuando acompañen a éste. No se debe dejar ningún espacio antes de la llamada de nota, vaya ésta tras signo de puntuación o tras cualquier letra.

Las ilustraciones, si las hubiera, se entregarán en formato tiff - con una resolución de 300 ppp - por separado con sus pies, indicando cuál ha de ser su ubicación aproximada en el texto. Se aportará la información pertinente acerca de su procedencia y sobre la propiedad de las imágenes. Para el resalte o grafismo enfático se evitará el uso de negritas, mayúsculas y subrayados y se usarán cursivas. En relación con los distintos apartados y subapartados, se evitarán en la medida de lo posible numeraciones innecesarias (el cuerpo de la letra, su estilo y su ubicación en la página reflejarán adecuadamente la jerarquía de los epígrafes).

Las citas bibliográficas se integrarán en el texto principal del artículo mediante el sistema Harvard según el modelo (Apellido del autor, año: páginas), por ejemplo: (Lacarra, 1972: 13-18). Las entradas del apartado bibliográfico del final del artículo se compondrán siguiendo las pautas que se indican a continuación:

\section{Artículo de revista:}

APELlidos del autor, Nombre (año), «Título del artículo», Título de la revista, volumen, número: páginas.

\section{Ejemplo:}

LACARRA DE Miguel, José María (1979), «Introducción al estudio de los mudéjares aragoneses», Aragón en la Edad Media, 2: 7-22.

\section{Libro completo:}

APELLIDOS del autor, Nombre (año), Título. Lugar de edición, Editorial. 


\section{Ejemplo:}

LACARRA DE Miguel, José María (1972), Aragón en el pasado. Madrid, EspasaCalpe.

\section{Capítulo de libro:}

APELlidos del autor, Nombre (año), «Título del capítulo.» En Nombre y Apellido del responsable de edición del libro, mención de responsabilidad en abreviatura: coord., dir., ed., Título del libro. Lugar de edición, Editorial: páginas.

\section{Ejemplo:}

SÁNCHEZ, Manuel (2010), «La monarquía y las ciudades desde el observatorio de la fiscalidad.» En José Ángel Sesma, coord., La Corona de Aragón en el centro de su historia, 1208-1458. La Monarquía aragonesa y los reinos de la Corona. Zaragoza, Grupo de Investigación CEMA: 45-66.

\section{Por último, los originales habrán de atenerse a los siguientes criterios.}

- En las abreviaturas se situará el punto antes de la letra volada (ejemplos: M. $^{\mathrm{a}}, \mathrm{n}^{\circ}{ }^{\circ}$ ).

- No se confundirán abreviaturas con símbolos: éstos no llevan punto al final ni marca de plural (km, g, h).

- No se separarán las letras de las siglas entre sí mediante puntos ni espacios. En plural las siglas son invariables (los PC, las ONG, los ISBN).

- Las palabras se separarán con un solo espacio, nunca con dos o más.

- Figurarán en cursiva todas las palabras - excepto nombres propios - y expresiones aisladas que no estén en el idioma principal del artículo.

- Figurarán con tilde y con diéresis las mayúsculas que las exijan, en castellano o en cualquier otro idioma.

- No se dejará espacio antes de los signos de puntuación simples (punto, coma, dos puntos, punto y coma, puntos suspensivos) ni antes de la primera palabra ni después de la última de un texto entrecomillado, entre paréntesis, entre corchetes, entre signos de interrogación o exclamación y entre rayas.

- La direcciones WEB se escriben entre signos landas. Ejemplo: $<$ https://papiro.unizar.es/ojs/index.php/aem>

- La raya o guion largo (-), de uso equivalente al de un paréntesis, debe distinguirse perfectamente del guion corto (-).

- No añadir punto tras cierre de interrogación o exclamación ni, por descontado, tras los puntos suspensivos, que siempre serán tres.

- Como comillas principales se usarán las latinas («»).

- Si una parte de un texto entre comillas latinas («») fuese entrecomillado, se emplean las inglesas (“").

- Cuando se define o traduce una voz, esta última se escribe en cursiva y la definición/traducción entre comillas simples (' ') y en redonda. 
- Si hay apéndice documental, los documentos irán numerados en caracteres arábigos, y en cada uno de ellos se hará constar la fecha (año, mes y día) y lugar, el regesto y la signatura.

- Las referencias a Archivos se darán desarrolladas la primera vez que aparecen. Después se utilizarán las siglas, sin puntos entre ellas (ACA, AHN).

- Cuando se cita un folio (f.) o folios (ff.) de un manuscrito o impreso, deberá especificarse si se trata del recto (f. 1 o 1 r) o del verso (f. 1v). 


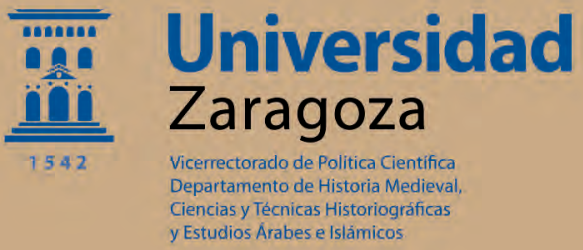

